\title{
Switching Controller Design for a Class of Markovian Jump Nonlinear Systems Using Stochastic Small-Gain Theorem
}

\author{
Jin Zhu, ${ }^{1}$ Junhong Park, ${ }^{1}$ Kwan-Soo Lee, $^{1}$ and Maksym Spiryagin ${ }^{2}$ \\ ${ }^{1}$ Department of Mechanical Engineering, Hanyang University, 133-791 Seoul, South Korea \\ 2 Dipartimento di Meccanica, Politecnico di Torino, 10129 Torino, Italy \\ Correspondence should be addressed to Jin Zhu, jinzhu@ustc.edu
}

Received 2 October 2008; Revised 24 December 2008; Accepted 16 January 2009

Recommended by Leonid Shaikhet

\begin{abstract}
Switching controller design for a class of Markovian jump nonlinear systems with unmodeled dynamics is considered in this paper. Based on the differential equation and infinitesimal generator of jump systems, the concept of Jump Input-to-State practical Stability (JISpS) in probability and stochastic Lyapunov stability criterion are put forward. By using backsetpping technology and stochastic small-gain theorem, a switching controller is proposed which ensures JISpS in probability for the jump nonlinear system. A simulation example illustrates the validity of this design.
\end{abstract}

Copyright (C) 2009 Jin Zhu et al. This is an open access article distributed under the Creative Commons Attribution License, which permits unrestricted use, distribution, and reproduction in any medium, provided the original work is properly cited.

\section{Introduction}

Stability of dynamic systems has been the primary study topic for system analysis. After Lyapunov's second method was created in 1892, it has been developed and applied by many researchers in the past century with fruitful classical stability results achieved. Among these important developments is the Input-to-State Stability (ISS) property, which was firstly formulated by Sontag [1] and has found wide use in engineering by incorporating the idea of nonlinear small-gain theorem [2,3]. The ISS-based small-gain theorem has some advantages over the earlier passivity-based small-gain theorem and is currently becoming a desirable tool for nonlinear stability analysis, especially in the case of nonlinear robust stabilization for systems with nonlinear uncertainties and unmodeled dynamics [4, 5]. Among all the practical nonlinear systems with uncertainties, the systems of lower-triangular form are of great importance; such systems have several special properties so that they are recently attracting great attention. Firstly, the "lower-triangular" form has close connection with feedback linearzation method; therefore this provides convenience to designers. Second, 
many real-world dynamic systems are of lower-triangular form [6, 7], and some general systems can be transformed to lower-triangular form via mathematical method [8]. For this reason, lower-triangle nonlinear system can find its wide applications in lots of practical dynamic systems: turbine generator and water turbing generator [9], intelligent robot [10] and missile [11], and so forth. However, dynamic processes in this field are very difficult to describe exactly and depend on many factors. For example, imagining an attacking missile tracking a moving target, this dynamic process is a classical problem of model following and tracking; till now, different control algorithms have been put forward by using ideal assumptions $[12,13]$. However, the missile itself may have variable structures subject to random changes and/or failures of its components or environments during its flight; such problems also occur in the case of the moving of robots or the operation of generators. Therefore, an urgent requirement appears to remodel such dynamic processes to meet the need of accuracy and precision.

On the other hand, Markovian jump systems, which were firstly put forward by Kac and Krasovskii [14], have now become convenient tools for representing many realworld systems $[15,16]$ and therefore arouse much research attention in recent years. In the case of fault detection, fault-tolerant control, and multimodal control, discrete jumps in the continuous dynamics are used to model component failures and sudden switch of system dynamics. With further study of Markovian jump systems, many achievements have been made in the last decade, among which Shaikhet and Mao performed foundational work on stochastic stability for jump systems [17-19] and jump systems with time delays [20-23]. Based on the stochastic stability, more efforts are devoted to applications of jump system model: system state estimation [24, 25], controller design [26-28], and hierarchical reinforcement learning for model-free jump systems [29, 30]. However, in the referred works concerned with controller design problems, assumptions are firstly made that system models considered only consist of static uncertainty. This is an ideal approximation of real situation and not the case nevertheless. As we know, Markovian jump systems are used to represent a class of systems which are usually accompanied by sudden change of working environment or system dynamics. For this reason, practical jump systems are usually accompanied by uncertainties, and it is hard to describe these uncertainties, with precise mathematical model. Thus, how to stabilize Markovian jump systems with unmodeled dynamic uncertainties is a concernful work in our view.

In this paper, we focus on the switching controller design for a class of Markovian jump nonlinear systems with dynamic uncertainties. The control strategy ensures robustness property of systems in the presentence of dynamic uncertainties. And our main contributions are composed of three aspects.

(i) Stochastic differential equation for Markovian jump system is given according to generalized Itô formula, and the similar result is achieved by Yuan and Mao [19] with a different method. Based on differential equation, the martingale process caused by Markovian process is incarnated in the procedure of controller design by applying mathematical transform.

(ii) We introduce the concept of Jump Input-to-State practical Stability (JISpS) and give stochastic Lyapunov stability criterion.

(iii) By composing backstepping technology and stochastic small-gain theorem, a switching controller is proposed. It is shown that all signals of the closed-loop system are globally uniformly ultimately bounded. And the closed-loop system is JISpS in probability. 
The rest of this paper is organized as follows. Section 2 begins with some mathematical notions and Markovian jump system model along with its differential equation. In Section 3, we introduce the notion of JISpS and stochastic Lyapunov stability criterion. Section 4 presents the problem description. In Section 5, a switching controller is given based on backstepping technology and stochastic small-gain theorem. In Section 6, an example is shown to illustrate the validity of the design. Finally, conclusions are drawn in Section 7.

\section{Stochastic Differential Equation of Markovian Jump System}

Throughout the paper, unless otherwise specified, we denote by $\left(\Omega, \mathcal{F},\left\{\mathcal{F}_{t}\right\}_{t \geq 0}, P\right)$ a complete probability space with a filtration $\left\{\mathcal{F}_{t}\right\}_{t \geq 0}$ satisfying the usual conditions (i.e., it is right continuous, and $\mp_{0}$ contains all $p$-null sets). Let $|x|$ stand for the usual Euclidean norm for a vector $x$ and $\left\|x_{t}\right\|$ stand for the supremum of vector $x$ over time period $\left[t_{0}, t\right]$, that is, $\left\|x_{t}\right\|=\sup _{t_{0} \leq s \leq t}|x(s)|$. The superscript $T$ will denote transpose, and we refer to $\operatorname{Tr}(\cdot)$ as the trace for matrix. In addition, we use $L_{2}(P)$ to denote the space of Lebesgue square integrable vector.

Take into account the following Markovian jump nonlinear system:

$$
d x=f(x, u, t, r(t)) d t+g(x, u, t, r(t)) d \omega(t),
$$

where $x \in \mathbb{R}^{n}$, and $u \in \mathbb{R}^{m}$ is the state vector and input vector of the system, respectively. $r(t), t \geq 0$ is a right-continuous Markov chain on the probability space taking values in finite state space $S=\{1,2, \ldots, N\}$, and $\omega(t)$ is $l$-dimensional independent standard Wiener process defined on the probability space, which is independent of the Markov chain $r(t)$. The functions $f: \mathbb{R}^{n+m} \times \mathbb{R}_{+} \times S \rightarrow \mathbb{R}^{n}$ and $g: \mathbb{R}^{n+m} \times \mathbb{R}_{+} \times S \rightarrow \mathbb{R}^{n \times l}$ are locally Lipschitz in $(x, u, k) \in \mathbb{R}^{n+m} \times S$, for all $t \geq 0$, namely, for any $h>0$, there is a constant $K_{h} \geq 0$ such that

$$
\begin{array}{r}
\left|f\left(x_{1}, u_{1}, t, k\right)-f\left(x_{2}, u_{2}, t, k\right)\right| \vee\left|g\left(x_{1}, u_{1}, t, k\right)-g\left(x_{2}, u_{2}, t, k\right)\right| \leq K_{h}\left(\left|x_{1}-x_{2}\right|+\left|u_{1}-u_{2}\right|\right), \\
\forall\left(x_{1}, u_{1}, t, k\right),\left(x_{2}, u_{2}, t, k\right) \in \mathbb{R}^{n+m} \times \mathbb{R}_{+} \times S, \quad\left|x_{1}\right| \vee\left|x_{2}\right| \vee\left|u_{1}\right| \vee\left|u_{2}\right| \leq h .
\end{array}
$$

Consider the right-continuous Markov chain $r(t)$, and we introduce $\Phi(t)=\left[\Phi_{1}(t), \Phi_{2}(t), \ldots\right.$, $\left.\Phi_{N}(t)\right]^{T}$, the indicator process for the regime $r(t)$, as

$$
\Phi_{j}(t)= \begin{cases}1, & r(t)=j \\ 0, & r(t) \neq j, j=1,2, \ldots, N\end{cases}
$$

And $\Phi(t)$ satisfies the following differential equation [15]:

$$
\Phi(t)=\Phi(0)+\Pi \int_{0}^{t} \Phi(s) d s+M(t)
$$


with $M(t)=\left[M_{1}(t), M_{2}(t), \ldots, M_{N}(t)\right]^{T}$, an $\mathcal{F}_{t}$-martingale satisfying $M(t) \in L^{2}(P)$, and $\Pi=\left[\pi_{k j}\right]_{N \times N}$ the chain generator, an $N \times N$ matrix. The entries $\pi_{k j}, k, j=1,2, \ldots, N$ are interpreted as transition rates such that

$$
P(r(t+d t)=j \mid r(t)=k)= \begin{cases}\pi_{k j} d t+o(d t), & \text { if } k \neq j \\ 1+\pi_{k j} d t+o(d t), & \text { if } k=j\end{cases}
$$

where $d t>0$ and $o(d t)$ satisfies $\lim _{d t \rightarrow 0}(o(d t) / d t)=0$. Here $\pi_{k j}>0(k \neq j)$ is the transition rate from regime $k$ to regime $j$. Notice that the total probability axiom imposes $\pi_{k k}$ negative and

$$
\sum_{j=1}^{N} \pi_{k j}=0, \quad \forall k \in S
$$

Let $C^{2,1}\left(\mathbb{R}^{n} \times \mathbb{R}_{+} \times S\right)$ denote the family of all functions $F(x, t, k)$ on $\mathbb{R}^{n} \times \mathbb{R}_{+} \times S$ which are continuously twice differentiable in $x$ and once in $t$. Furthermore, we will give the stochastic differentiable equation of $F(x, t, k)$.

Fix any $\left(x_{0}, t_{0}, k\right) \in \mathbb{R}^{n} \times \mathbb{R}_{+} \times S$; by the generalized Itô formula, we have

$$
\begin{aligned}
F(x, t, r(t))= & F\left(x_{0}, t_{0}, k\right)+\int_{t_{0}}^{t} \frac{\partial F(x, s, r(s))}{\partial s} d s+\int_{t_{0}}^{t} \frac{\partial F(x, s, r(s))}{\partial x} f(x, u, s, r(s)) d s \\
& +\int_{t_{0}}^{t} \frac{1}{2} \operatorname{Tr}\left[g^{T}(x, u, s, r(s)) \frac{\partial^{2} F(x, s, r(s))}{\partial x^{2}} g(x, u, s, r(s))\right] d s \\
& +\int_{t_{0}}^{t} \frac{\partial F(x, s, r(s))}{\partial x} g(x, u, s, r(s)) d \omega(s) \\
& +\int_{t_{0}}^{t} \sum_{j=1}^{N}[F(x, s, j)-F(x, s, k)] d \Phi_{j}(s) .
\end{aligned}
$$

According to (2.4), the differential equation of the indicator $\Phi(t)$ is as following:

$$
d \Phi(t)=\Pi \Phi(t) d t+d M(t) .
$$

Submit (2.8) into (2.7) and notice that

$$
\sum_{j=1}^{N} \pi_{k j} F(x, t, k)=0, \quad \forall k \in S
$$


There is

$$
\begin{aligned}
F(x, t, r(t))= & F\left(x_{0}, t_{0}, k\right)+\int_{t_{0}}^{t} \frac{\partial F(x, s, r(s))}{\partial s} d s+\int_{t_{0}}^{t} \frac{\partial F(x, s, r(s))}{\partial x} f(x, u, s, r(s)) d s \\
& +\int_{t_{0}}^{t} \frac{1}{2} \operatorname{Tr}\left[g^{T}(x, u, s, r(s)) \frac{\partial^{2} F(x, s, r(s))}{\partial x^{2}} g(x, u, s, r(s))\right] d s \\
& +\int_{t_{0}}^{t} \frac{\partial F(x, s, r(s))}{\partial x} g(x, u, s, r(s)) d \omega(s) \\
& +\int_{t_{0}}^{t} \sum_{j=1}^{N} \pi_{k j} F(x, s, j) d s+\int_{t_{0}}^{t} \sum_{j=1}^{N}[F(x, s, j)-F(x, s, k)] d M_{j}(s) .
\end{aligned}
$$

Therefore, the stochastic differentiable equation of $F(x, t, k)$ is given by the following:

$$
\begin{aligned}
d F(x, t, k)= & \frac{\partial F(x, t, k)}{\partial t} d t+\frac{\partial F(x, t, k)}{\partial x} f(x, u, t, k) d t \\
& +\frac{1}{2} \operatorname{Tr}\left[g^{T}(x, u, t, k) \frac{\partial^{2} F(x, t, k)}{\partial x^{2}} g(x, u, t, k)\right] d t \\
& +\sum_{j=1}^{N} \pi_{k j} F(x, t, j) d t+\frac{\partial F(x, t, k)}{\partial x} g(x, u, t, k) d \omega(t) \\
& +\sum_{j=1}^{N}[F(x, t, j)-F(x, t, k)] d M_{j}(t) .
\end{aligned}
$$

We take the expectation in (2.11), so that the the infinitesimal generator produces $[18,19,23]$

$$
\begin{aligned}
\mathcal{L} F(x, t, k)= & \frac{\partial F(x, t, k)}{\partial t}+\frac{\partial F(x, t, k)}{\partial x} f(x, u, t, k)+\sum_{j=1}^{N} \pi_{k j} F(x, t, j) \\
& +\frac{1}{2} \operatorname{Tr}\left[g^{T}(x, u, t, k) \frac{\partial^{2} F(x, t, k)}{\partial x^{2}} g(x, u, t, k)\right] .
\end{aligned}
$$

Remark 2.1. The differential equation of Markovian jump system (2.1) is given as above and, the similar result is also achieved by Yuan and Mao [19]. Compared with the differential equation of general nonjump systems, two parts come forth as differences: transition rates $\pi_{k j}$ and the martingale process $M(t)$, which are both caused by the property of Markov chain $r(t)$ (see (2.4)). Up till now, switching controller design for jump systems contains only the transition rate $\pi_{k j}$ in most cases. In this paper, the controller design will take into account the martingale process as well since the jump systems considered here are of the form of lower triangular. The detailed description will be given in Section 4. 


\section{JISpS and Stochastic Small-Gain Theorem}

Definition 3.1. Markovian jump system (2.1) is $p$-moment Jump Input-to-State practically Stable (JISpS) if there exist $\mathcal{K} \perp$ function $\beta(\cdot, \cdot), \mathcal{K}_{\infty}$ function $\gamma(\cdot)$, and a constant $d_{c} \geq 0$ such that

$$
E|x(t, k)|^{p} \leq \beta\left(\left|x_{0}\right|, t\right)+\gamma\left(\left\|u_{t}(k)\right\|\right)+d_{c}, \quad \forall t \geq 0, k \in S, x_{0} \in \mathbb{R}^{n} \backslash\{0\}, p>0 .
$$

Definition 3.2. Markovian jump system (2.1) is JISpS in probability if for any given $\epsilon>0$ there exist $\mathcal{K} \perp$ function $\beta(\cdot, \cdot), \mathcal{K}_{\infty}$ function $\gamma(\cdot)$, and a constant $d_{c} \geq 0$ such that

$$
P\left\{|x(t, k)|<\beta\left(\left|x_{0}\right|, t\right)+\gamma\left(\left\|u_{t}(k)\right\|\right)+d_{c}\right\} \geq 1-\epsilon, \quad \forall t \geq 0, k \in S, x_{0} \in \mathbb{R}^{n} \backslash\{0\} .
$$

Remark 3.3. The concept of Input-to-State Stability (ISS) is a well-known classical tool for designing nonlinear systems, which means for a bounded control input $u$, the trajectories remain in the ball of radius as $\beta\left(\left|x_{0}\right|, 0\right)+\gamma(\|u\|)$; furthermore, as time $t$ increases, all trajectories approach the smaller ball of radius $\gamma(\|u\|)$. However, for general nonlinear systems disturbed by noise and/or unmodeled dynamics, it is impossible to obtain such strong conclusion, therefore some generalized results are put forward: Noise-to-State Stability (NSS) [31] and Input-to-State practically Stable (ISpS) [32]. In the definition of ISpS, the trajectories remain in the ball of radius as $\beta\left(\left|x_{0}\right|, 0\right)+\gamma(\|u\|)+d_{c}$ instead of $\beta\left(\left|x_{0}\right|, 0\right)+\gamma(\|u\|)$. Similar to ISS, as time $t$ increases, all trajectories approach the smaller ball of radius $\gamma(\|u\|)+d_{c}$, and the system is still BIBO. As can be seen in the following analysis of this paper, bound $d_{c}$ can be made as small as possible by choosing appropriate control parameters. For some special cases, if $d_{c} \equiv 0$, the ISpS is reduced to ISS.

Remark 3.4. The definition of Input-to-State practically Stable (ISpS) in probability for nonjump stochastic system is put forward by Wu et al. [32], and the difference between JISpS in probability and ISpS in probability lies in the expressions of system state $x(t, k)$ and control signal $u_{t}(k)$. For nonjump system, system state and control signal contain only continuous time $t$ with $k \equiv 1$, while for jump system, they concern with both continuous time $t$ and discrete regime $k$. For different system dynamic $r(t)=k$, control signal $u_{t}(k)$ will differ even with the same time $t$ taken and that is the reason why it is called a switching controller. Based on the idea of switching control, the corresponding stability is called "Jump ISpS" and it is a more general extension of ISpS. By choosing $k \equiv 1$, the definition of JISpS will degenerate to ISpS.

Remark 3.5. This paper introduces two kinds of JISpS in the sense of stochastic stability: $p$ moment JISpS and JISpS in probability. According to the knowledge of stochastic process, if one system is $p$-moment stochastically stable, it must be stochastically stable in probability by using martingale inequality. Here only sufficient conditions for $p$-moment stochastic stability are considered and now introduce the following stochastic Lyapunov stability criterion. 
Theorem 3.6. For Markovian jump system (2.1), let $\alpha_{1}, \alpha_{2}, \lambda$, and $p$ be positive numbers. Assume that there exist a function $V(x, t, r(t)) \in C^{2,1}\left(\mathbb{R}^{n} \times \mathbb{R}_{+} \times S ; \mathbb{R}_{+}\right)$, a $\mathcal{K}_{\infty}$ function $X(\cdot)$, and constants $d, \varpi \geq 0$, satisfying

$$
\begin{aligned}
& \alpha_{1}|x|^{p} \leq V(x, t, k) \leq \alpha_{2}|x|^{p}+\varpi,
\end{aligned}
$$

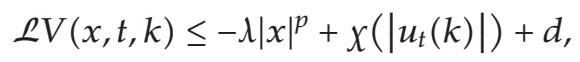

for all $(x, t, k) \in \mathbb{R}^{n} \times \mathbb{R}_{+} \times S$, then jump system (2.1) is pth moment JISpS and JISpS in probability as well.

Proof. Clearly the conclusion holds if $x_{0}=0$. So we only need proof for $x_{0} \neq 0$. Fix such $x_{0}$ arbitrarily, we write $x\left(t, k, x_{0}\right)$ as $x(t)$.

For each integer $h \geq 1$, define a stopping time as

$$
\tau_{h}=\inf \{t \geq 0:|x(t)| \geq h\}
$$

Obviously, $\tau_{h} \rightarrow \infty$ almost surely as $h \rightarrow \infty$. Noticing that $0<|x(t)| \leq h$ if $0 \leq t<\tau_{h}$, we can apply the generalized Itô formula to derive that for any $t \geq 0$

$$
\begin{aligned}
& E\left[e^{\left(\lambda / \alpha_{2}\right)\left(t \wedge \tau_{h}\right)} V\left(x\left(t \wedge \tau_{h}\right), t \wedge \tau_{h}, r\left(t \wedge \tau_{h}\right)\right)\right]
\end{aligned}
$$

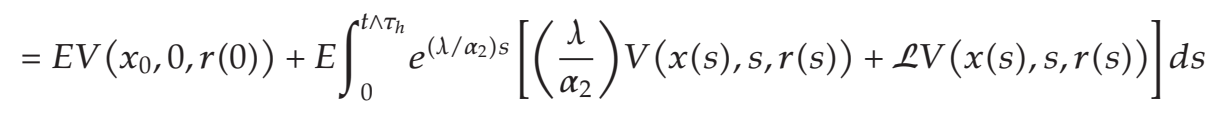

$$
\begin{aligned}
& \leq E V\left(x_{0}, 0, r(0)\right)+E \int_{0}^{t \wedge \tau_{h}} e^{\left(\lambda / \alpha_{2}\right) s}\left[\left(\frac{\lambda}{\alpha_{2}}\right)\left(\alpha_{2}|x|^{p}+\varpi\right)-\lambda|x|^{p}+x\left(\left|u_{s}(r(s))\right|\right)+d\right] d s \\
& =E V\left(x_{0}, 0, r(0)\right)+E \int_{0}^{t \wedge \tau_{h}} e^{\left(\lambda / \alpha_{2}\right) s}\left[X\left(\left|u_{s}(r(s))\right|\right)+\frac{\lambda}{\alpha_{2}} \varpi+d\right] d s
\end{aligned}
$$

Let $h \rightarrow \infty$, apply Fatou's lemma to (3.6), and we have

$$
E\left[e^{\left(\lambda / \alpha_{2}\right) t} V(x(t), t, r(t))\right] \leq E V\left(x_{0}, 0, r(0)\right)+E \int_{0}^{t} e^{\left(\lambda / \alpha_{2}\right) s}\left[x\left(\left|u_{s}(r(s))\right|\right)+\frac{\lambda}{\alpha_{2}} \varpi+d\right] d s .
$$

According to Mean value theorems for integration, there is

$$
\begin{aligned}
E\left[e^{\left(\lambda / \alpha_{2}\right) t} V(x(t), t, k)\right] & =e^{\left(\lambda / \alpha_{2}\right) t} E V(x(t), t, k) \\
& \leq E V\left(x_{0}, 0, r(0)\right)+E\left\{\sup _{0 \leq s \leq t}\left[X\left(\left|u_{s}(k)\right|\right)+\frac{\lambda}{\alpha_{2}} \varpi+d\right]\right\} \cdot \int_{0}^{t} e^{\left(\lambda / \alpha_{2}\right) s} d s .
\end{aligned}
$$


Noticing the property of $\mathcal{K}_{\infty}$ function, the following inequality is deduced:

$$
\begin{aligned}
e^{\left(\lambda / \alpha_{2}\right) t} E V(x(t), t, k) & \leq E V\left(x_{0}, 0, r(0)\right)+\left\{x\left(\sup _{0 \leq s \leq t}\left|u_{s}(k)\right|\right)+\frac{\lambda}{\alpha_{2}} \varpi+d\right\} \cdot \int_{0}^{t} e^{\left(\lambda / \alpha_{2}\right) s} d s \\
& =E V\left(x_{0}, 0, r(0)\right)+\left\{x\left(\left\|u_{t}(k)\right\|\right)+\frac{\lambda}{\alpha_{2}} \varpi+d\right\} \cdot\left(\frac{\alpha_{2}}{\lambda}\right)\left(e^{\left(\lambda / \alpha_{2}\right) t}-1\right) \\
& \leq E V\left(x_{0}, 0, r(0)\right)+\left\{x\left(\left\|u_{t}(k)\right\|\right)+\frac{\lambda}{\alpha_{2}} \varpi+d\right\} \cdot\left(\frac{\alpha_{2}}{\lambda}\right) e^{\left(\lambda / \alpha_{2}\right) t} .
\end{aligned}
$$

Submitting (3.3) into (3.9) gives

$$
e^{\left(\lambda / \alpha_{2}\right) t} \cdot \alpha_{1} E|x|^{p} \leq E V\left(x_{0}, 0, r(0)\right)+\left\{x\left(\left\|u_{t}(k)\right\|\right)+\frac{\lambda}{\alpha_{2}} \varpi+d\right\} \cdot\left(\frac{\alpha_{2}}{\lambda}\right) e^{\left(\lambda / \alpha_{2}\right) t}
$$

Consequently,

$$
\begin{aligned}
E|x|^{p} & \leq \frac{1}{\alpha_{1}} e^{-\left(\lambda / \alpha_{2}\right) t} E V\left(x_{0}, 0, r(0)\right)+\frac{\alpha_{2}}{\lambda \alpha_{1}}\left\{x\left(\left\|u_{t}(k)\right\|\right)+\frac{\lambda}{\alpha_{2}} \varpi+d\right\} \\
& \leq \frac{\alpha_{2}}{\alpha_{1}} e^{-\left(\lambda / \alpha_{2}\right) t}\left|x_{0}\right|^{p}+\frac{\alpha_{2}}{\lambda \alpha_{1}} x\left(\left\|u_{t}(k)\right\|\right)+\frac{\alpha_{2}}{\lambda \alpha_{1}} d+\frac{1}{\alpha_{1}} \varpi\left(1+e^{-\left(\lambda / \alpha_{2}\right) t}\right) \\
& \leq \frac{\alpha_{2}}{\alpha_{1}} e^{-\left(\lambda / \alpha_{2}\right) t}\left|x_{0}\right|^{p}+\frac{\alpha_{2}}{\lambda \alpha_{1}} x\left(\left\|u_{t}(k)\right\|\right)+\frac{\alpha_{2}}{\lambda \alpha_{1}} d+\frac{2}{\alpha_{1}} \varpi .
\end{aligned}
$$

In (3.11), define $\mathcal{K} \perp$ function $\beta(\cdot, \cdot), \mathcal{K}_{\infty}$ function $\gamma(\cdot)$, and positive constant $d_{c}$ as:

$$
\beta\left(\left|x_{0}\right|, t\right)=\frac{\alpha_{2}}{\alpha_{1}} e^{-\left(\lambda / \alpha_{2}\right) t}\left|x_{0}\right|^{p}, \quad \gamma\left(\left\|u_{t}(k)\right\|\right)=\frac{\alpha_{2}}{\lambda \alpha_{1}} x\left(\left\|u_{t}(k)\right\|\right), \quad d_{c}=\frac{\alpha_{2}}{\lambda \alpha_{1}} d+\frac{2}{\alpha_{1}} \varpi .
$$

There is

$$
E|x(t, k)|^{p} \leq \beta\left(\left|x_{0}\right|, t\right)+\gamma\left(\left\|u_{t}(k)\right\|\right)+d_{c}
$$

This completes the proof.

Consider the jump interconnected dynamic system described in Figure 1:

$$
\begin{aligned}
& d x_{1}=f_{1}\left(x_{1}, x_{2}, \Xi_{1}, r(t)\right) d t+g_{1}\left(x_{1}, x_{2}, \Xi_{1}, r(t)\right) d W_{t 1}, \\
& d x_{2}=f_{2}\left(x_{1}, x_{2}, \Xi_{2}, r(t)\right) d t+g_{2}\left(x_{1}, x_{2}, \Xi_{2}, r(t)\right) d W_{t 2},
\end{aligned}
$$

where $x=\left(x_{1}^{T}, x_{2}^{T}\right)^{T} \in \mathbb{R}^{n_{1}+n_{2}}$ is the state of system, and $\Xi_{i}(i=1,2)$ denotes exterior disturbance and/or interior uncertainty. $W_{t i}$ is independent Wiener noise with appropriate dimension, and we introduce the following stochastic nonlinear small-gain theorem as a lemma, which is an extension of the corresponding result in Wu et al. [32]. 


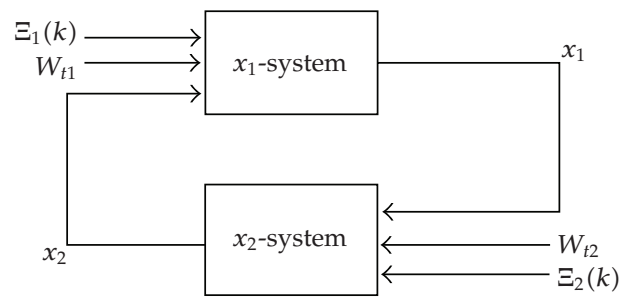

Figure 1: Interconnected feedback system.

Lemma 3.7 (Stochastic small-gain theorem). Suppose that both the $x_{1}$-system and $x_{2}$-system are JISpS in probability with $\left(\Xi_{1}(k), x_{2}(k)\right)$ as input and $x_{1}(k)$ as state, and $\left(\Xi_{2}(k), x_{1}(k)\right)$ as input and $x_{2}(k)$ as state, respectively, that is, for any given $\epsilon_{1}, \epsilon_{2}>0$,

$$
\begin{aligned}
& P\left\{\left|x_{1}(t, k)\right|<\beta_{1}\left(\left|x_{1}(0, k)\right|, t\right)+\gamma_{1}\left(\left\|x_{2}(t, k)\right\|\right)+\gamma_{w 1}\left(\left\|\Xi_{1 t}(k)\right\|\right)+d_{1}\right\} \geq 1-\epsilon_{1}, \\
& P\left\{\left|x_{2}(t, k)\right|<\beta_{2}\left(\left|x_{2}(0, k)\right|, t\right)+\gamma_{2}\left(\left\|x_{1}(t, k)\right\|\right)+\gamma_{w 2}\left(\left\|\Xi_{2 t}(k)\right\|\right)+d_{2}\right\} \geq 1-\epsilon_{2}
\end{aligned}
$$

hold with $\beta_{i}(\cdot, \cdot)$ being $\mathcal{K} \perp$ function, $\gamma_{i}$ and $\gamma_{w i}$ being $\mathcal{K}_{\infty}$ functions, and $d_{i}$ being nonnegative constants, $i=1,2$. If there exist nonnegative parameters $\rho_{1}, \rho_{2}, s_{0}$ such that nonlinear gain functions $\gamma_{1}, \gamma_{2}$ satisfy

$$
\left(1+\rho_{1}\right) \gamma_{1} \circ\left(1+\rho_{2}\right) \gamma_{2}(s) \leq s, \quad \forall s \geq s_{0}
$$

the interconnected system is JISpS in probability with $\Xi(k)=\left(\Xi_{1}(k), \Xi_{2}(k)\right)$ as input and $x=$ $\left(x_{1}, x_{2}\right)$ as state, that is, for any given $\epsilon>0$, there exist a $\mathcal{\mathcal { L }} \mathcal{\perp}$ function $\beta_{c}(\cdot, \cdot)$, a $\boldsymbol{\mathcal { K }}_{\infty}$ function $\gamma_{w}(\cdot)$, and a parameter $d_{c} \geq 0$ such that

$$
P\left\{|x(t, k)|<\beta_{c}\left(\left|x_{0}\right|, t\right)+\gamma_{w}\left(\left\|\Xi_{t}(k)\right\|\right)+d_{c}\right\} \geq 1-\epsilon .
$$

Remark 3.8. Small-gain theorem for nonlinear systems was firstly provided by Mareels and Hill [33] and was extended to the stochastic case by $\mathrm{Wu}$ et al. [32]. The above stochastic small-gain theorem for jump systems is an extension of the nonjump case. This extension can be achieved without any mathematical difficulties, and the proof process is the same as in [32]. The reason is that in Lemma 3.7 we only take into account the interconnection relationships between interconnected system and its subsystems, despite subsystems are of jump or nonjump case. If both subsystems are nonjump and ISpS in probability, respectively, the interconnected system is ISpS in probability. In contrast, if both subsystems are jump and JISpS in probability, respectively, the interconnected system is JISpS in probability and so on. 


\section{Problem Description}

Consider the following Markovian jump nonlinear systems with unmodeled dynamics described by

$$
\begin{gathered}
d \xi=q(y, \xi, t, r(t)) d t, \\
d x_{i}=x_{i+1} d t+f_{i}\left(X_{i}, t, r(t)\right) d t+\Delta_{i}(X, \xi, t, r(t)) d t, \quad i=1,2, \ldots, n-1 \\
d x_{n}=u d t+f_{n}(X, t, r(t)) d t+\Delta_{n}(X, \xi, t, r(t)) d t \\
y=x_{1},
\end{gathered}
$$

where $X_{i}=\left(x_{1}, x_{2}, \ldots, x_{i}\right)^{T} \in \mathbb{R}^{i}\left(X \in \mathbb{R}^{n}\right)$ is system state vector, $u \in \mathbb{R}$ is system input signal, $\xi \in \mathbb{R}^{n_{0}}$ is unmeasured state vector, and $y$ is output signal. The Markov chain $r(t) \in S$ is as defined in Section 2. $f_{i}: \mathbb{R}^{i} \times \mathbb{R}_{+} \times S \rightarrow \mathbb{R}$ is a smooth function, and $\Delta_{i}(X, \xi, t, r(t))$ denotes the unmodeled dynamic uncertainty which could be different with different system regime $r(t)$. Both $f_{i}(\cdot, \cdot, \cdot)$ and $\Delta_{i}(\cdot, \cdot, \cdot, \cdot)$ are locally Lipschitz as described in Section 2.

Our design purpose is to find a switching controller $u$ of the form $u(x, t, k), k \in S$, such that the closed-loop jump system could be JISpS in probability, and the system output $y$ could be within an attractive region around the equilibrium point with radius as small as possible. In this paper, the following assumptions are made for jump system (4.1):

(A1) The $\xi$ subsystem with input $y$ is JISpS in probability, namely, there exists a smooth positive definite Lyapunov function $V_{0}(\xi, t, k) \in C^{2,1}\left(\mathbb{R}^{n_{0}} \times \mathbb{R}_{+} \times S ; \mathbb{R}_{+}\right)$such that

$$
\begin{gathered}
\alpha_{10}|\xi|^{p_{0}} \leq V_{0}(\xi, t, k) \leq \alpha_{20}|\xi|^{p_{0}}, \\
\mathcal{L} V_{0}(\xi, t, k) \leq-\lambda_{0}|\xi|^{p_{0}}+\chi_{0}(|y|)+d_{0},
\end{gathered}
$$

where $x_{0}(\cdot)$ is $\mathcal{K}_{\infty}$ function, $p_{0}$ is positive integer, and $\alpha_{10}, \alpha_{20}, \lambda_{0}>0, d_{0} \geq 0$ are constants.

(A2) For each $i=1,2, \ldots, n, k \in S$, there exists an unknown positive constant $p_{i}^{*} \leq \bar{p}_{i}$ such that

$$
\left|\Delta_{i}(X, \xi, t, k)\right| \leq p_{i}^{*} \bar{\phi}_{i 1}\left(X_{i}, k\right)+p_{i}^{*} \bar{\phi}_{i 2}(|\xi|, k)
$$

where $\bar{p}_{i}$ is known constant and $\bar{\phi}_{i 1}(\cdot, k), \bar{\phi}_{i 2}(\cdot, k)$ are known nonnegative smooth functions for any given $k \in S$.

For the design of switching controller, we introduce the following lemmas.

Lemma 4.1 (Young's inequality). For any two vectors $x, y \in \mathbb{R}^{n}$, the following inequality holds

$$
x^{T} y \leq \frac{\epsilon^{p}}{p}|x|^{p}+\frac{1}{q \epsilon^{q}}|y|^{q},
$$

where $\epsilon>0$ and the constants $p>1, q>1$ satisfy $(p-1)(q-1)=1$. 
Lemma 4.2 (Martingale representation [34]). Let $B(t)=\left[B_{1}(t), B_{2}(t), \ldots, B_{N}(t)\right]$ be $N$ dimensional standard Wiener noise. Suppose $M(t)$ is an $\mathcal{F}_{t}^{N}$-martingale (w.r.t. $P$ ) and that $M(t) \in$ $L^{2}(P)$, for all $t \geq 0$, then there exists a stochastic process $\Psi(t) \in L^{2}(P)$, such that

$$
d M(t)=\Psi(t) \cdot d B(t)
$$

\section{Controller Design and Stability Analysis}

Now we seek the switching controller $u(x, t, k)$ for jump system (4.1) so that the closed-loop system could be JISpS in probability. Perform a new transformation as

$$
z_{i}=x_{i}-\alpha_{i-1}\left(X_{i-1}, t, k\right), \quad \forall i=1,2, \ldots, n, k \in S
$$

For simplicity, we just denote $\alpha_{i-1}\left(X_{i-1}, t, k\right), f_{i}\left(X_{i}, t, k\right), \Delta_{i}(X, \xi, t, k), q(y, \xi, t, k)$ by $\alpha_{i-1}(k)$, $f_{i}(k), \Delta_{i}(k), q(k)$, where $\alpha_{0}(k)=0, \alpha_{n}(k)=u(x, t, k), \forall k \in S$, and the new coordinate is $Z=\left(z_{1}, z_{2}, \ldots, z_{n}\right)$.

According to stochastic differential equation (2.11), one has:

$$
\begin{aligned}
d z_{i}= & d x_{i}-d \alpha_{i-1}(k) \\
= & {\left[x_{i+1}+f_{i}(k)+\Delta_{i}(k)\right] d t-\frac{\partial \alpha_{i-1}(k)}{\partial t} d t-\sum_{j=1}^{i-1} \frac{\partial \alpha_{i-1}(k)}{\partial x_{j}}\left[x_{j+1}+f_{j}(k)+\Delta_{j}(k)\right] d t } \\
& -\sum_{j=1}^{N} \pi_{k j} \alpha_{i-1}(j) d t+\sum_{j=1}^{N}\left[\alpha_{i-1}(k)-\alpha_{i-1}(j)\right] d M_{j}(t) \\
= & {\left[z_{i+1}+\alpha_{i}(k)+f_{i}(k)+\Lambda_{i}(k)\right] d t-\frac{\partial \alpha_{i-1}(k)}{\partial t} d t-\sum_{j=1}^{i-1} \frac{\partial \alpha_{i-1}(k)}{\partial x_{j}}\left[x_{j+1}+f_{j}(k)\right] d t } \\
& -\sum_{j=1}^{N} \pi_{k j} \alpha_{i-1}(j) d t+\Gamma_{i}(k) d M(t) .
\end{aligned}
$$

Here we define

$$
\begin{gathered}
\Lambda_{i}(k) \triangleq \Delta_{i}(k)-\sum_{j=1}^{i-1} \frac{\partial \alpha_{i-1}(k)}{\partial x_{j}} \Delta_{j}(k) \\
\Gamma_{i}(k) \triangleq\left[\alpha_{i-1}(k)-\alpha_{i-1}(1), \alpha_{i-1}(k)-\alpha_{i-1}(2), \ldots, \alpha_{i-1}(k)-\alpha_{i-1}(N)\right] .
\end{gathered}
$$

From assumption (A2), one gets that there exist nonnegative smooth functions $\phi_{i 1}(\cdot, k)$, $\phi_{i 2}(\cdot, k)$ satisfying

$$
\left|\Lambda_{i}(k)\right| \leq p_{i}^{*} \phi_{i 1}\left(X_{i}, k\right)+p_{i}^{*} \phi_{i 2}(|\xi|, k)
$$

The inequality (5.4) could easily be deduced by using Lemma 4.1 . 
Now we turn to the martingale process $M(t)$; according to Lemma 4.2 , there exist a function $\Psi(t) \in L^{2}(P)$ and an $N$-dimensional standard Wiener noise $B(t)$ satisfying $d M(t)=$ $\Psi(t) d B(t)$, where $E\left[\Psi(t) \Psi(t)^{T}\right]=\psi(t) \psi(t)^{T} \leq Q<\infty$, and $Q$ is a positive bounded constant. Therefore we have

$$
\begin{aligned}
d z_{i}=\left\{z_{i+1}+\alpha_{i}(k)+f_{i}(k)+\Lambda_{i}(k)-\frac{\partial \alpha_{i-1}(k)}{\partial t}\right. & \\
& \left.\quad-\sum_{j=1}^{i-1} \frac{\partial \alpha_{i-1}(k)}{\partial x_{j}}\left[x_{j+1}+f_{j}(k)\right]-\sum_{j=1}^{N} \pi_{k j} \alpha_{i-1}(j)\right\} d t+\Gamma_{i}(k) \Psi(t) d B(t) .
\end{aligned}
$$

Remark 5.1. Differential equation of new coordinate $Z(t)$ is deduced as above. The martingale process resulting from Markov process is transformed into Wiener noise by using Martingale representation theorem, and it will affect the Lyapunov function construction and affect the remainder of the control design process; for nonjump systems with uncertainty, a quadratic Lyapunov is chosen to meet control performance in most cases [32, 35, 36]. However, for jump systems, this choice will fail because of the existence of martingale process (or Wiener noise). To solve this problem, we suggest using quartic Lyapunov function instead of quadratic one, and this will increase largely the difficulty of design.

Choose the quartic Lyapunov function as

$$
V=\frac{1}{4} \sum_{i=1}^{n} z_{i}^{4}
$$

In the view of (5.5) and (5.6), the infinitesimal generator of $V$ satisfies

$$
\begin{aligned}
\mathcal{L} V=\sum_{i=1}^{n} z_{i}^{3}\left\{z_{i+1}+\alpha_{i}(k)+f_{i}(k)+\Lambda_{i}(k)-\frac{\partial \alpha_{i-1}(k)}{\partial t}\right. & \\
& \left.\quad-\sum_{j=1}^{i-1} \frac{\partial \alpha_{i-1}(k)}{\partial x_{j}}\left[x_{j+1}+f_{j}(k)\right]-\sum_{j=1}^{N} \pi_{k j} \alpha_{i-1}(j)\right\}+\frac{3}{2} \sum_{i=1}^{n} z_{i}^{2} \Gamma_{i}(k) \psi \psi^{T} \Gamma_{i}^{T}(k) \\
\leq \sum_{i=1}^{n} z_{i}^{3}\left\{\left(\frac{3}{4} \delta_{i}^{4 / 3}+\frac{1}{4 \delta_{i-1}^{4}}\right) z_{i}+\alpha_{i}(k)+f_{i}(k)+\Lambda_{i}(k)-\frac{\partial \alpha_{i-1}(k)}{\partial t}\right. & \\
& \left.\quad-\sum_{j=1}^{i-1} \frac{\partial \alpha_{i-1}(k)}{\partial x_{j}}\left[x_{j+1}+f_{j}(k)\right]-\sum_{j=1}^{N} \pi_{k j} \alpha_{i-1}(j)+\mu z_{i}\left[\Gamma_{i}(k) \Gamma_{i}^{T}(k)\right]^{2}\right\}+\frac{9 n}{16 \mu} Q^{2} .
\end{aligned}
$$


The following inequalities could be deduced by using Young's inequality and norm inequalities with the help of changing the order of summations or exchanging the indices of the summations:

$$
\begin{aligned}
\sum_{i=1}^{n} z_{i}^{3} z_{i+1} & \leq \frac{3}{4} \sum_{i=1}^{n-1} \delta_{i}^{4 / 3} z_{i}^{4}+\frac{1}{4} \sum_{i=1}^{n-1} \frac{1}{\delta_{i}^{4}} z_{i+1}^{4} \\
& =\sum_{i=1}^{n}\left(\frac{3}{4} \delta_{i}^{4 / 3}+\frac{1}{4 \delta_{i-1}^{4}}\right) z_{i}^{4} \\
\frac{3}{2} \sum_{i=1}^{n} z_{i}^{2} \Gamma_{i}(k) \psi \psi^{T} \Gamma_{i}^{T}(k) & \leq \frac{3}{2} \sum_{i=1}^{n} z_{i}^{2} \Gamma_{i}(k) Q \Gamma_{i}^{T}(k) \\
& \leq \sum_{i=1}^{n} \mu z_{i}^{4}\left[\Gamma_{i}(k) \Gamma_{i}^{T}(k)\right]^{2}+\sum_{i=1}^{n} \frac{9}{16 \mu} Q^{2} \\
& =\sum_{i=1}^{n} \mu z_{i}^{4}\left[\Gamma_{i}(k) \Gamma_{i}^{T}(k)\right]^{2}+\frac{9 n}{16 \mu} Q^{2},
\end{aligned}
$$

where $\delta_{0}=\infty, \delta_{n}=0$, and $\mu>0, \delta_{i}>0, i=1,2, \ldots, n$ are design parameters.

Based on assumption (A2) and (5.4), we obtain the following inequality by applying Lemma 4.1:

$$
\begin{aligned}
z_{i}^{3} \Lambda_{i}(k) & \leq\left|z_{i}^{3} \Lambda_{i}(k)\right| \\
& \leq\left|z_{i}^{3}\right| *\left[p_{i}^{*} \phi_{i 1}\left(X_{i}, k\right)+p_{i}^{*} \phi_{i 2}(|\xi|, k)\right] \\
& =\left|z_{i}^{3}\right| p_{i}^{*} \phi_{i 1}\left(X_{i}, k\right)+\left|z_{i}^{3}\right| p_{i}^{*} \phi_{i 2}(|\xi|, k) \\
& \leq\left[\bar{p}_{i} z_{i}^{3} \widehat{\phi}_{i 1}\left(X_{i}, k\right)+\sigma_{i}\right]+\left[z_{i}^{6} \bar{p}_{i}^{2}+\frac{1}{4} \phi_{i 2}^{2}(|\xi|, k)\right] .
\end{aligned}
$$

Here $\widehat{\phi}_{i 1}\left(X_{i}, k\right)=\left(\bar{p}_{i} / 4 \sigma_{i}\right) z_{i}^{3} \phi_{i}^{2}\left(X_{i}, k\right), \sigma_{i}>0$ are design parameters.

Submit (5.9) into (5.7), there is

$$
\begin{aligned}
\mathcal{L} V \leq \sum_{i=1}^{n} z_{i}^{3}\{( & \left.\frac{3}{4} \delta_{i}^{4 / 3}+\frac{1}{4 \delta_{i-1}^{4}}\right) z_{i}+\alpha_{i}(k)+f_{i}(k)+\bar{p}_{i} \widehat{\phi}_{i 1}\left(X_{i}, k\right)+\bar{p}_{i}^{2} z_{i}^{3}-\frac{\partial \alpha_{i-1}(k)}{\partial t} \\
& \left.\quad-\sum_{j=1}^{i-1} \frac{\partial \alpha_{i-1}(k)}{\partial x_{j}}\left[x_{j+1}+f_{j}(k)\right]-\sum_{j=1}^{N} \pi_{k j} \alpha_{i-1}(j)+\mu z_{i}\left[\Gamma_{i}(k) \Gamma_{i}^{T}(k)\right]^{2}\right\} \\
& +\sum_{i=1}^{n} \frac{1}{4} \phi_{i}^{2}(|\xi|, k)+\sum_{i=1}^{n} \sigma_{i}+\frac{9 n}{16 \mu} Q^{2} .
\end{aligned}
$$


Choose the virtual control signal as

$$
\begin{aligned}
\alpha_{i}(k)=- & c_{i} z_{i}-\left(\frac{3}{4} \delta_{i}^{4 / 3}+\frac{1}{4 \delta_{i-1}^{4}}\right) z_{i}-f_{i}(k)-\bar{p}_{i} \widehat{\phi}_{i 1}\left(X_{i}, k\right)-\bar{p}_{i}^{2} z_{i}^{3}+\frac{\partial \alpha_{i-1}(k)}{\partial t} \\
& +\sum_{j=1}^{i-1} \frac{\partial \alpha_{i-1}(k)}{\partial x_{j}}\left[x_{j+1}+f_{j}(k)\right]+\sum_{j=1}^{N} \pi_{k j} \alpha_{i-1}(j)-\mu z_{i}\left[\Gamma_{i}(k) \Gamma_{i}^{T}(k)\right]^{2} .
\end{aligned}
$$

Thus the real control signal $u$ is $u(x, t, k)=\alpha_{n}(k)$ such that

$$
\begin{aligned}
\varrho V & \leq-\sum_{i=1}^{n} c_{i} z_{i}^{4}+\sum_{i=1}^{n} \frac{1}{4} \phi_{i}^{2}(|\xi|, k)+\sum_{i=1}^{n} \sigma_{i}+\frac{9 n}{16 \mu} Q^{2} \\
& \leq-c V+V_{\xi}(|\xi|, k)+d_{z},
\end{aligned}
$$

where $c=\min \left\{4 c_{i}\right\}, d_{z}=\sum_{i=1}^{n} \sigma_{i}+(9 n / 16 \mu) Q^{2}$, and $\mathcal{K}_{\infty}$ function $V_{\xi}$ is chosen to satisfy $V_{\xi}(|\xi|, k) \geq \sum_{i=1}^{n}(1 / 4) \phi_{i}^{2}(|\xi|, k)$.

Theorem 5.2. If Assumptions (A1) and (A2) hold and a switching control law (5.11) is adopted, the interconnected Markovian jump system (4.1) is JISpS in probability, and all solutions of closedloop system are ultimately bounded. Furthermore, the system output could be regulated to a small neighborhood of the equilibrium point with preset precision in probability within finite time.

Proof. From Assumption (A1), the $\xi$ subsystem is JISpS in probability. There exist $\alpha_{10}, \alpha_{20}, p_{0}>$ 0 such that

$$
\alpha_{10}|\xi|^{p_{0}} \leq V_{0}(\xi, t, k) \leq \alpha_{20}|\xi|^{p_{0}}
$$

Considering (5.12), for any given $0<D_{1}<c$, there is

$$
\mathcal{L} V \leq-\left(c-D_{1}\right) V+V_{\xi}(|\xi|, k)+d_{z}-D_{1} V .
$$

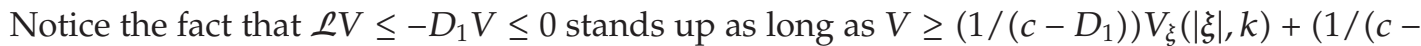
$\left.\left.D_{1}\right)\right) d_{z}$ and vice versa. Thus we have

$$
\begin{aligned}
P\{V & \left.\leq \max \left\{\frac{3}{c-D_{1}} V_{\xi}\left[\left\|\frac{V_{0}(\xi, t, k)}{\alpha_{10}}\right\|^{1 / p_{0}}\right], \frac{3 d_{z}}{c-D_{1}}\right\}\right\} \\
& \geq P\left\{\left\{\rho V \leq-\iota_{1} D_{1} V\right\} \cap\left\{V \leq \max \left\{\frac{3}{c-D_{1}} V_{\xi}\left[\left\|\frac{V_{0}(\xi, t, k)}{\alpha_{10}}\right\|^{1 / p_{0}}\right], \frac{3 d_{z}}{c-D_{1}}\right\}\right\}\right\} \\
& \geq P\left\{V \leq \max \left\{e^{-\iota_{1} D_{1} t} V(0), \frac{3}{c-D_{1}} V_{\xi}\left[\left\|\frac{V_{0}(\xi, t, k)}{\alpha_{10}}\right\|^{1 / p_{0}}\right], \frac{3 d_{z}}{c-D_{1}}\right\}\right\} \\
& \geq P\left\{V \leq \frac{1}{3} e^{-\iota_{1} D_{1} t} V(0)+\frac{1}{c-D_{1}} V_{\xi}\left[\left\|\frac{V_{0}(\xi, t, k)}{\alpha_{10}}\right\|^{1 / p_{0}}\right]+\frac{d_{z}}{c-D_{1}}\right\} .
\end{aligned}
$$


In (5.15), appropriate parameter $\iota_{1}$ can be chosen to satisfy $e^{-\iota_{1} D_{1} t} V(0) \leq \max \{(3 /(c-$ $\left.\left.\left.D_{1}\right)\right) V_{\xi}\left[\left\|V_{0}(\xi, t, k) / \alpha_{10}\right\|^{1 / p_{0}}\right], 3 d_{z} /\left(c-D_{1}\right)\right\}$.

According to Theorem 3.6 and (5.12), with switching controller $u(k)$ adopted, the $X$ subsystem of jump system (4.1) is JISpS in probability with $X_{i}$ as system state and $\xi$ as input, which means for any given $\epsilon_{1}>0$, there exists $\mathcal{K} \mathcal{L}$ function $\beta_{1}(\cdot, \cdot)=(1 / 3) e^{-t_{1} D_{1} t} V(0), \mathcal{K}_{\infty}$ function $\gamma_{1}\left(\left\|V_{0}(k)\right\|\right)=\left(1 /\left(c-D_{1}\right)\right) V_{\xi}\left[\left\|V_{0}(\xi, t, k) / \alpha_{10}\right\|^{1 / p_{0}}\right]$, and $d_{1}=3 d_{z} /\left(c-D_{1}\right)$ such that

$$
\begin{aligned}
P\left\{\frac{1}{4}|y|^{4}=\frac{1}{4}\left|Z_{1}\right|^{4} \leq V \leq \max \left\{\frac{3}{c-D_{1}} V_{\xi}\left[\left\|\frac{V_{0}(\xi, t, k)}{\alpha_{10}}\right\|^{1 / p_{0}}\right], \frac{3 d_{z}}{c-D_{1}}\right\}\right\} \\
\geq P\left\{|V| \leq \beta_{1}(V(0), t)+\gamma_{1}\left(\left\|V_{0}(k)\right\|\right)+d_{1}\right\} \\
\geq 1-\epsilon_{1} .
\end{aligned}
$$

On the other hand, according to Assumption (A1), there is

$$
\mathcal{L} V_{0}(\xi, t, k) \leq-\lambda_{0}|\xi|^{p_{0}}+\chi_{0}(|y|)+d_{0} \leq-\frac{\lambda_{0}}{\alpha_{20}} V_{0}(\xi, t, k)+\chi_{0}\left(|4 V(k)|^{1 / 4}\right)+d_{0}
$$

Similarly, by choosing parameter $0<D_{2}<\lambda_{0} / \alpha_{20}$, for any given $\epsilon_{2}>0$, there exist $\mathcal{L} \mathcal{L}$ function $\beta_{2}(\cdot, \cdot)=(1 / 3) e^{-t_{2} D_{2} t} V_{0}\left(\xi_{0}, 0, k\right), \mathcal{K}_{\infty}$ function $\gamma_{2}(\|V(k)\|)=\left(3 \alpha_{20} /\left(\lambda_{0}-\right.\right.$ $\left.D_{2} \alpha_{20}\right) \chi_{0}\left(\|4 V(k)\|^{1 / 4}\right)$, and $d_{2}=3 d_{0} \alpha_{20} /\left(\lambda_{0}-D_{2} \alpha_{20}\right)$ such that

$$
\begin{aligned}
& P\left\{V_{0}(\cdot, \cdot, k) \leq \max \left\{\frac{3}{\lambda_{0} / \alpha_{20}-D_{2}} x_{0}\left(\|4 V(k)\|^{1 / 4}\right), \frac{3 d_{0}}{\lambda_{0} / \alpha_{20}-D_{2}}\right\}\right\} \\
& \quad \geq P\left\{\left|V_{0}\right| \leq \beta_{2}\left(V_{0}(0), t\right)+\gamma_{2}(\|V(k)\|)+d_{2}\right\} \\
& \quad \geq 1-\epsilon_{2} .
\end{aligned}
$$

Here parameter $\iota_{2}$ can be chosen to satisfy $e^{-t_{2} D_{2} t} V_{0}\left(\xi_{0}, 0, k\right) \leq \max \left\{\left(3 /\left(\lambda_{0} / \alpha_{20}\right)-\right.\right.$ $\left.\left.D_{2}\right) \chi_{0}\left(\|4 V(k)\|^{1 / 4}\right), 3 d_{0} /\left(\lambda_{0} / \alpha_{20}\right)-D_{2}\right\}$.

By combining (5.16) and (5.18) we choose parameters $D_{1}, D_{2}, \rho_{1}, \rho_{2}$ guaranteeing that

$$
\left(1+\rho_{1}\right) \gamma_{1} \circ\left(1+\rho_{2}\right) \gamma_{2}(s) \leq s, \quad \forall s \geq 0
$$

According to stochastic small-gain theorem, for any given $\epsilon>0$, there exists $\mathcal{K} \perp$ function $\beta_{c}$ such that

$$
P\left\{\left|V_{\Xi}(t, k)\right|<\beta_{c}\left(\left|V_{\Xi}(0)\right|, t\right)+d_{c}\right\} \geq 1-\epsilon,
$$

where $V_{\Xi} \triangleq\left(V, V_{0}\right)^{T}, d_{c} \triangleq\left(1+\rho_{1}^{-1}\right)\left[d_{1}+\gamma_{1}\left(\left(1+\rho_{2}^{-1}\right) d_{2}\right)\right]+\left(1+\rho_{2}^{-2}\right)\left[d_{2}+\gamma_{2}\left(\left(1+\rho_{1}^{-1}\right) d_{1}\right)\right]$ is given as in [32]. From (5.20) it can be seen that all solutions of closed-loop system are ultimately bounded in probability.

According to (5.20) and the property of $\mathcal{K} \perp$ function, for any given $\delta_{1}>0$, there exists $T>0$. If $t>T$, there is $\beta_{c}\left(\left|V_{\Xi}(0)\right|, t\right)<\delta_{1}$. At the same time by choosing approximate parameter, it can be guaranteed that $d_{c}<\delta_{1}$. 


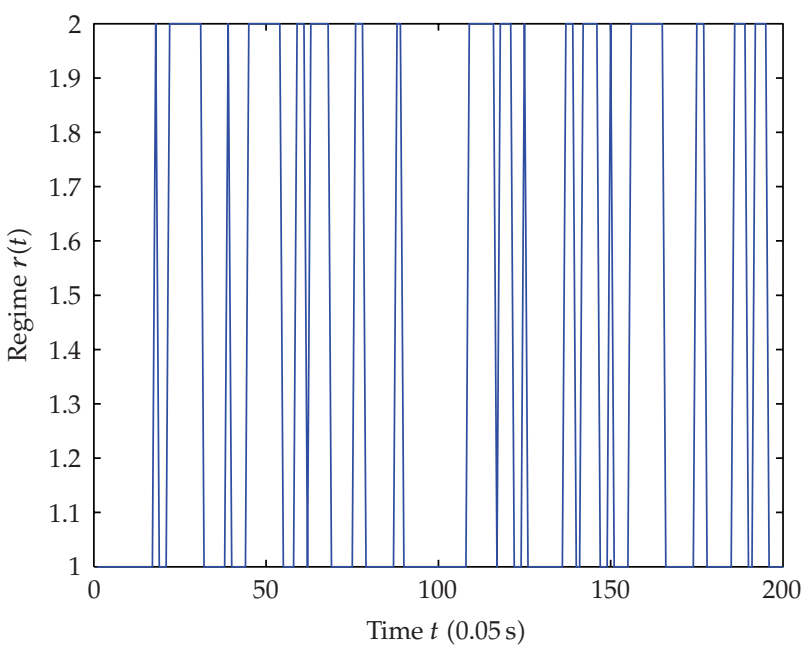

Figure 2: Regime transition $r(t)$.

Let $\delta=2 \delta_{1}$, thus we have that for any given $\epsilon>0$, there exists $T>0$ and $\delta>0$ such that if $t>T$, the output of jump system $y$ satisfies

$$
P\{|y(t)|<\delta\} \geq 1-\epsilon,
$$

meanwhile $\delta$ can be made as small as possible by choosing approximate parameters $c_{i}, \sigma_{i}, D_{1}, D_{2}, \rho_{1}, \rho_{2}>0$. The proof is completed.

Remark 5.3. Theorem 5.2 shows that if both $X$ subsystem and $\xi$ subsystem are JISpS in probability, the jump system (4.1) is JISpS in probability with appropriate control parameters chosen. Meanwhile the system output can be regulated to a small region in probability with preset precision within finite time. In the following simulation, we will show how different parameters affect the system states and output.

\section{Simulation}

Consider a two-order Markovian jump nonlinear system with regime transition space $S=$ $\{1,2\}$, and the transition rate matrix is $\Pi=\left[\begin{array}{cc}\pi_{11} & \pi_{12} \\ \pi_{21} & \pi_{22}\end{array}\right]=\left[\begin{array}{cc}-3 & 3 \\ 4 & -4\end{array}\right]$.

The system with unmodeled dynamics is as follows:

$$
\begin{gathered}
d \xi=q\left(x_{1}, \xi, t, r(t)\right) d t \\
d x_{1}=x_{2} d t+f_{1}\left(x_{1}, t, r(t)\right) d t+\Delta_{1}(X, \xi, t, r(t)) d t \\
d x_{2}=u d t+f_{2}(X, t, r(t)) d t+\Delta_{2}(X, \xi, t, r(t)) d t \\
y=x_{1} .
\end{gathered}
$$




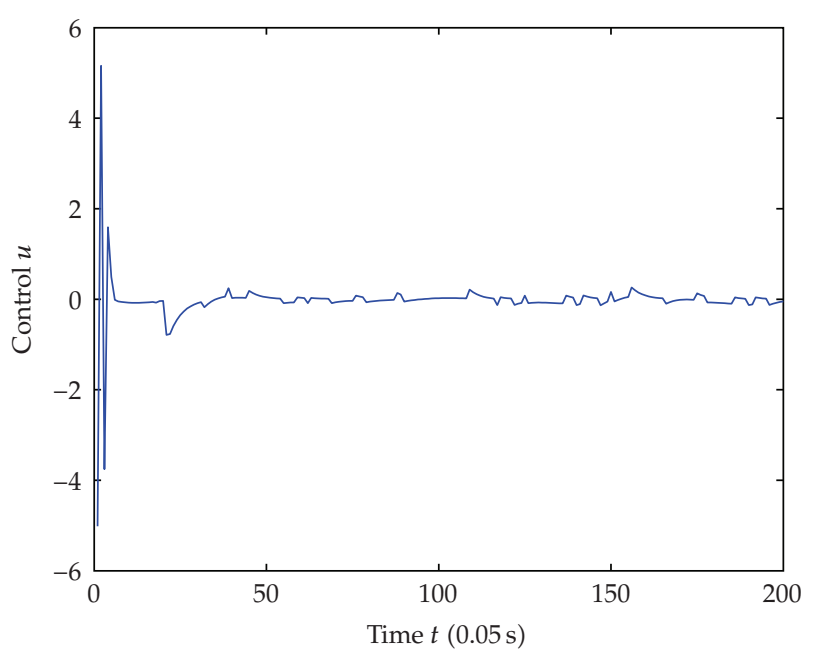

Figure 3: Switching controller $u$.

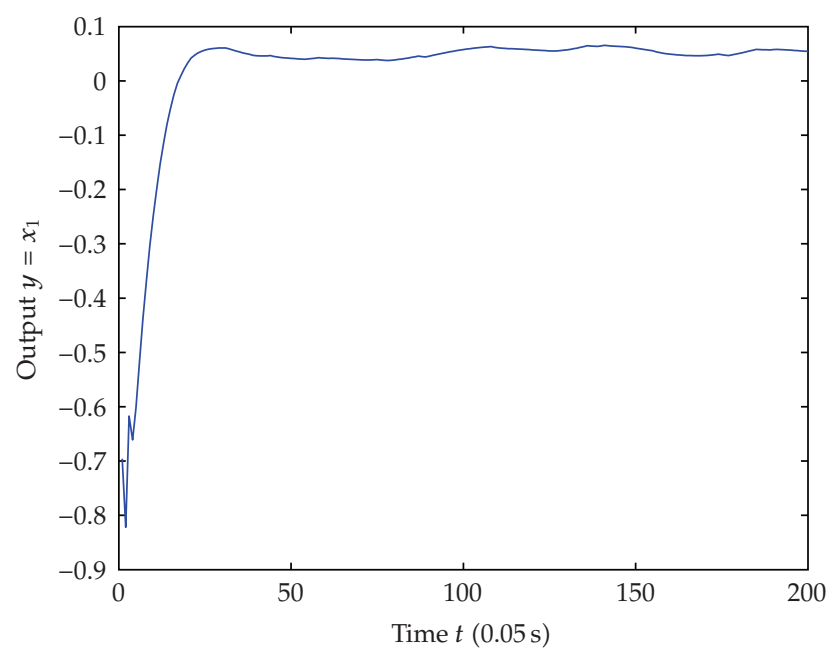

Figure 4: System output $y$.

Here

$$
\begin{gathered}
q\left(x_{1}, \xi, t, 1\right)=-\xi+0.2 x_{1}+0.5, \quad q\left(x_{1}, \xi, t, 2\right)=-\xi+0.3 x_{1} \sin t, \\
f_{1}\left(x_{1}, t, 1\right)=x_{1}^{2}, \quad f_{1}\left(x_{1}, t, 2\right)=x_{1}, \\
\Delta_{1}(X, \xi, t, 1)=0.3 \xi+0.2 x_{1} \sin (3 t), \quad \Delta_{1}(X, \xi, t, 2)=x_{1} \xi, \\
f_{2}(X, t, 1)=x_{1} \sin x_{2}, \quad f_{2}(X, t, 2)=x_{1}+x_{2}, \\
\Delta_{2}(X, \xi, t, 1)=0.2 \xi \cos (2 t)+0.1 x_{1}, \quad \Delta_{2}(X, \xi, t, 2)=x_{1}|\xi|^{1 / 2} .
\end{gathered}
$$




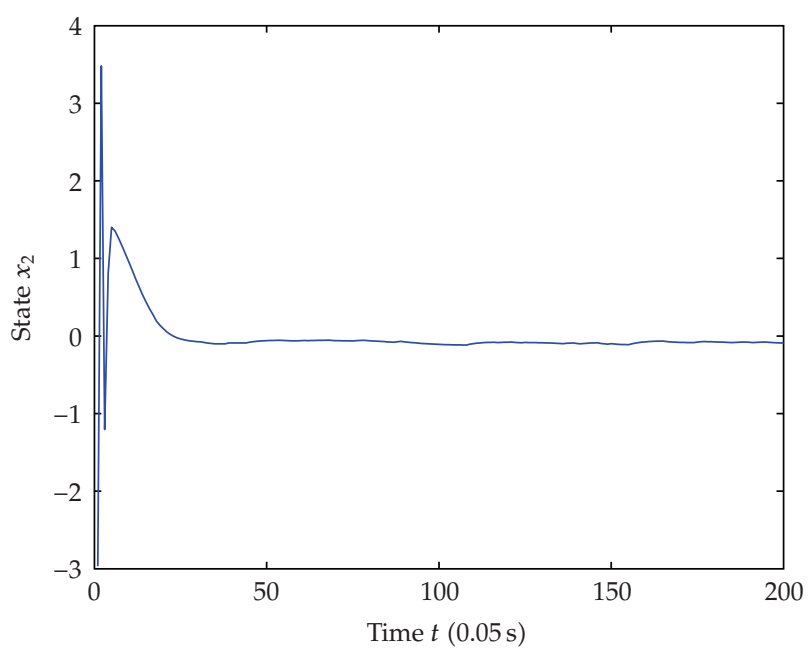

Figure 5: System state $x_{2}$.

From Assumption (A2), we have

$$
\begin{array}{ll}
\Delta_{1}(X, \xi, t, 1) \leq p_{1}^{*}|\xi|+p_{1}^{*}\left|x_{1}\right|, & \Delta_{1}(X, \xi, t, 2) \leq p_{1}^{*}|\xi|^{2}+p_{1}^{*}\left|x_{1}\right|^{2}, \\
\Delta_{2}(X, \xi, t, 1) \leq p_{2}^{*}|\xi|+p_{2}^{*}\left|x_{1}\right|, & \Delta_{2}(X, \xi, t, 2) \leq p_{2}^{*}|\xi|+p_{2}^{*}\left|x_{1}\right|^{2},
\end{array}
$$

where $p_{1}^{*} \leq 0.5, p_{2}^{*} \leq 0.5$, and the $\xi$ subsystem satisfies

$$
\mathcal{L} V_{0}(\xi, t, k) \leq-\frac{4}{10}|\xi|^{2}+x_{0}\left(\left|x_{1}\right|\right)+d_{0}
$$

where $V_{0}=(1 / 2) \xi^{2}, X_{0}\left(\left|x_{1}\right|\right)=0.15\left|x_{1}\right|^{2}, d_{0}=0.125$. Thus the control law is taken as follows (here $\delta_{1}=1$ ).

Case 1. The system regime is $k=1$ :

$$
\begin{aligned}
\alpha_{1}(1)= & -\left(c_{1}+\frac{3}{4}\right) x_{1}-x_{1}^{2}-\frac{1}{4} x_{1}^{3}-\frac{1}{16 \sigma_{1}} x_{1}^{5} \\
\alpha_{2}(1)= & -\left(c_{2}+\frac{1}{4}\right) z_{2}(1)-x_{1} \sin x_{2}-\frac{1}{16 \sigma_{2}} z_{2}^{3}(1) x_{1}^{2}-\frac{1}{4} z_{2}^{3}(1) \\
& -\left(c_{1}+\frac{3}{4}+2 x_{1}+\frac{3}{4} x_{1}^{2}+\frac{5}{16 \sigma_{1}} x_{1}^{4}\right)\left(x_{1}^{2}+x_{2}\right) \\
& +\pi_{11} \alpha_{1}(1)+\pi_{12} \alpha_{1}(2)-\mu z_{2}(1)\left[\alpha_{1}(1)-\alpha_{1}(2)\right]^{4} \\
z_{2}(1)= & x_{2}-\alpha_{1}(1)
\end{aligned}
$$




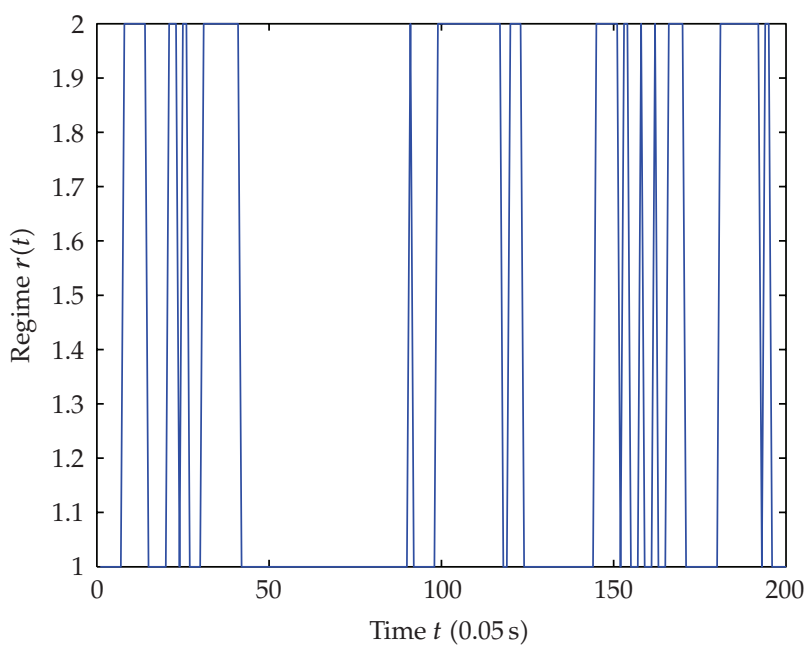

Figure 6: Regime transition $r(t)$.

Case 2. The system regime is $k=2$ :

$$
\begin{aligned}
\alpha_{1}(2)= & -\left(c_{1}+\frac{7}{4}\right) x_{1}-\frac{1}{4} x_{1}^{3}-\frac{1}{16 \sigma_{1}} x_{1}^{7} \\
\alpha_{2}(2)= & -\left(c_{2}+\frac{1}{4}\right) z_{2}(2)-\frac{1}{16 \sigma_{2}} z_{2}^{3}(2) x_{1}^{4}-\frac{1}{4} z_{2}^{3}(2)-\left(c_{1}+\frac{11}{4}+\frac{3}{4} x_{1}^{2}+\frac{7}{16 \sigma_{1}} x_{1}^{6}\right)\left(x_{1}+x_{2}\right) \\
& +\pi_{21} \alpha_{1}(1)+\pi_{22} \alpha_{1}(2)-\mu z_{2}(2)\left[\alpha_{1}(1)-\alpha_{1}(2)\right]^{4}, \\
z_{2}(2)= & x_{2}-\alpha_{1}(2) .
\end{aligned}
$$

In computation, we set the initial value to be $x_{1}=-0.9, x_{2}=3.3$ and the time step to be 0.05 second. For comparison, two groups of different control parameters are given. First, we take the parameter with values $c_{1}=c_{2}=2, \sigma_{1}=\sigma_{2}=1, \mu=5$, and the simulation results are as follows. Figure 2 shows the regime transition of the jump system, and Figure 3 shows the corresponding switching controller $u$. Figure 4 shows the system output $y$ which is defined as the system state $x_{1}$, and Figure 5 shows system state $x_{2}$.

Now we choose different control parameters as $c_{1}=c_{2}=6, \sigma_{1}=\sigma_{2}=0.5, \mu=10$ and repeat the simulation. The simulation results are as follows. Figure 6 shows the regime transition of the jump system, and Figure 7 shows the corresponding switching controller $u$. Figure 8 shows the system output $y$ which is defined as the system state $x_{1}$, and Figure 9 shows system state $x_{2}$.

Comparing the results from two simulations, all the signals of closed-loop system are globally uniformly ultimately bounded, and the system output can be regulated to a neighborhood near the equilibrium point despite of different experiment samples. As can be seen from the figures, larger values of $c_{1}, c_{2}$ help to increase the convergence speed of system states while larger value of $\mu$ and smaller values of $\sigma_{1}, \sigma_{2}$ help to increase the precision. If one wants the system states to converge to the neighborhood of the equilibrium point with 


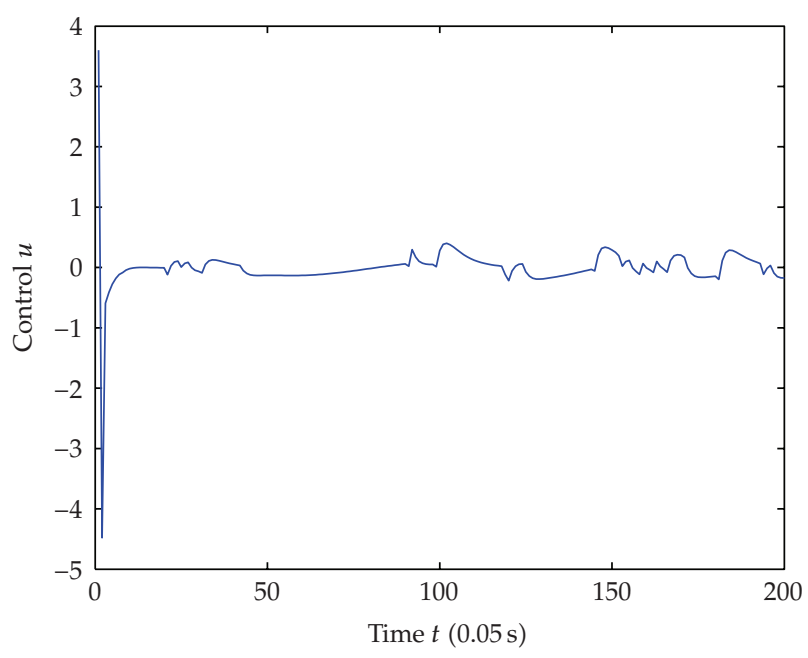

Figure 7: Switching controller $u$.

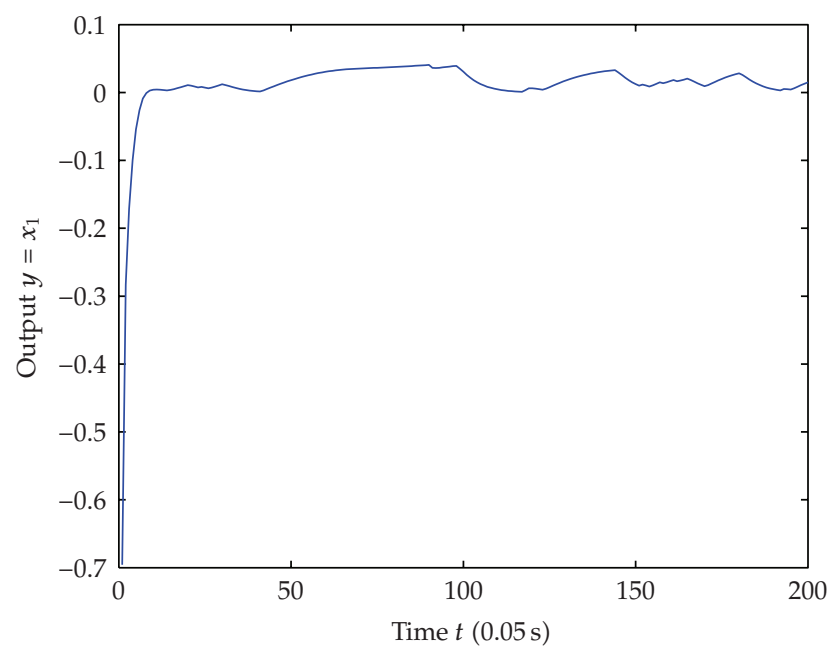

Figure 8: System output $y$.

fast speed and an acceptable precision, one should increase the value of $c_{1}, c_{2}, \mu$ and decrease $\sigma_{1}, \sigma_{2}$ though this choice will increase the cost of control signals.

Remark 6.1. Much research work has been performed toward the study of nonlinear system by using small-gain theorem $[4,32,33]$. In contrast to their contributions, this paper focuses on the switching controller design for Markovian jump nonlinear system which is a more general form of nonjump systems. For each different regime $r(t)=k$, the actual controller $u(k)$ is different, and it consists of two parts with obvious difference (see (5.11)): the coupling of regime $\pi_{k j} \alpha_{i-1}(j)$ and $\mu z_{i}\left[\Gamma_{i}(k) \Gamma_{i}^{T}(k)\right]^{2}$, which are both caused by the Markovian jumps (see (2.4)). By defining regime $k \equiv 1$, the above two terms will reduce to zero. Thus this switching controller design is capable of stabilizing the general nonjump system as well. 


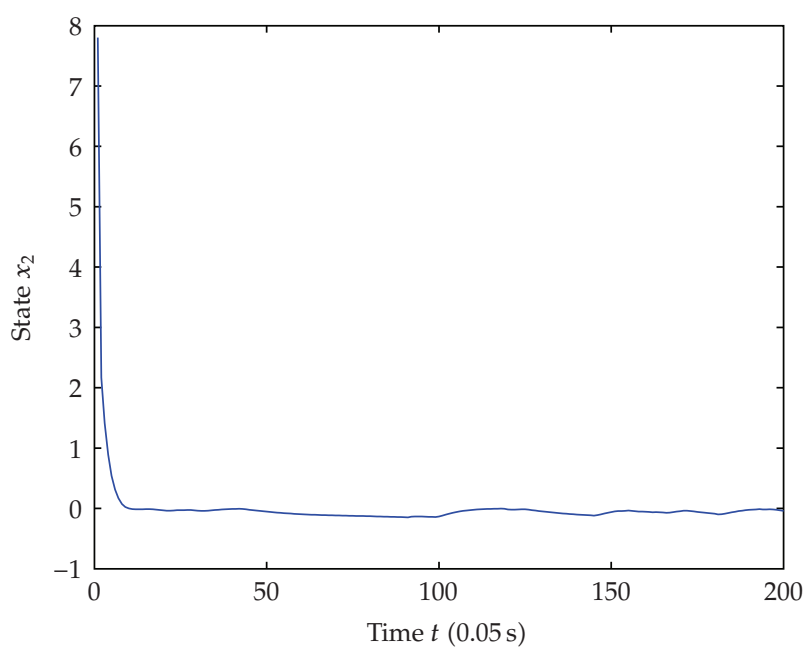

Figure 9: System state $x_{2}$.

\section{Conclusion}

In this paper, the authors take into account switching controller design for a class of Markovian jump nonlinear system with unmodeled dynamics. Based on the differential equation and infinitesimal generator of jump systems, the concept of Jump Input-to-State Stability (JISpS) and stochastic Lyapunov stability criterion are put forward. Moreover, the martingale process caused by the stochastic Markovian jumps is converted into Wiener noise. By using backstepping technology and stochastic small-gain theorem, a switching controller is proposed which ensures JISpS in probability of the jump nonlinear system. And system output can be regulated in probability to a small neighborhood of the equilibrium point with preset precision. The result presented in this paper also stands for the general nonjump system.

\section{Acknowledgment}

This work has been funded by BK21 research project: Switching Control of Systems with Structure Uncertainty and Noise.

\section{References}

[1] E. D. Sontag, "Smooth stabilization implies coprime factorization," IEEE Transactions on Automatic Control, vol. 34, no. 4, pp. 435-443, 1989.

[2] H. K. Khalil, Nonlinear Systems, Prentice-Hall, Englewood Cliffs, NJ, USA, 2nd edition, 1996.

[3] P. V. Kokotović and M. Arcak, "Constructive nonlinear control: a historical perspective," Automatica, vol. 37, no. 5, pp. 637-662, 2001.

[4] Z.-P. Jiang, "A combined backstepping and small-gain approach to adaptive output feedback control," Automatica, vol. 35, no. 6, pp. 1131-1139, 1999.

[5] Z.-P. Jiang, I. Mareels, and D. Hill, "Robust control of uncertain nonlinear systems via measurement feedback," IEEE Transactions on Automatic Control, vol. 44, no. 4, pp. 807-812, 1999.

[6] M. Krstić, I. Kanellakopoulos, and P. V. Kokotović, Nonlinear and Adaptive Control Design, John Wiley \& Sons, New York, NY, USA, 1995. 
[7] D. Seto, A. M. Annaswamy, and J. Baillieul, "Adaptive control of nonlinear systems with a triangular structure," IEEE Transactions on Automatic Control, vol. 39, no. 7, pp. 1411-1428, 1994.

[8] S. Čelikovský and H. Nijmeijer, "Equivalence of nonlinear systems to triangular form: the singular case," Systems $\mathcal{E}$ Control Letters, vol. 27, no. 3, pp. 135-144, 1996.

[9] H. Chen, H.-B. Ji, B. Wang, and H.-S. Xi, "Coordinated passivation techniques for the dual-excited and steam-valving control of synchronous generators," IEE Proceedings: Control Theory and Applications, vol. 153, no. 1, pp. 69-73, 2006.

[10] Z.-P. Jiang and H. Nijmeijer, "Tracking control of mobile robots: a case study in backstepping," Automatica, vol. 33, no. 7, pp. 1393-1399, 1997.

[11] K. D. Do, Z. P. Jiang, and J. Pan, “On global tracking control of a VTOL aircraft without velocity measurements," IEEE Transactions on Automatic Control, vol. 48, no. 12, pp. 2212-2217, 2003.

[12] Y.-C. Chang and H.-M. Yen, "Adaptive output feedback tracking control for a class of uncertain nonlinear systems using neural networks," IEEE Transactions on Systems, Man, and Cybernetics, Part B, vol. 35, no. 6, pp. 1311-1316, 2005.

[13] Z. Cai, M. S. de Queiroz, and D. M. Dawson, "Robust adaptive asymptotic tracking of nonlinear systems with additive disturbance," IEEE Transactions on Automatic Control, vol. 51, no. 3, pp. 524529, 2006.

[14] I. Ya. Kac and N. N. Krasovskii, "About stability of systems with stochastic parameters," Prikladnaya Matematika i Makhanika, vol. 24, no. 5, pp. 809-823, 1960.

[15] M. Mariton, Jump Linear Systems in Automatic Control, Marcel-Dekker, New York, NY, USA, 1990.

[16] I. Ya. Kac, "Method of Lyapunov functions in problems of stability and stabilization of systems of stochastic structure," Ekaterinburg, Russia, 1998.

[17] L. Shaikhet, "Stability of stochastic hereditary systems with Markovian switching," Theory of Stochastic Process, vol. 2, no. 18, pp. 180-184, 1996.

[18] X. Mao, "Stability of stochastic differential equations with Markovian switching," Stochastic Processes and Their Applications, vol. 79, no. 1, pp. 45-67, 1999.

[19] C. Yuan and X. Mao, "Asymptotic stability in distribution of stochastic differential equations with Markovian switching," Stochastic Processes and Their Applications, vol. 103, no. 2, pp. 277-291, 2003.

[20] X. Mao and L. Shaikhet, "Delay-dependent stability criteria for stochastic differential delay equations with Markovian switching," Stability and Control: Theory and Applications, vol. 3, no. 2, pp. 88-102, 2000.

[21] X. Mao, "Robustness of stability of stochastic differential delay equations with Markovian switching," Stability and Control: Theory and Applications, vol. 3, no. 1, pp. 48-61, 2000.

[22] X. Mao, "Asymptotic stability for stochastic differential delay equations with Markovian switching," Functional Differential Equations, vol. 9, no. 1-2, pp. 201-220, 2002.

[23] C. Yuan and X. Mao, "Robust stability and controllability of stochastic differential delay equations with Markovian switching," Automatica, vol. 40, no. 3, pp. 343-354, 2004.

[24] C. E. de Souza, A. Trofino, and K. A. Barbosa, "Mode-independent $\mathscr{l}_{\infty}$ filters for Markovian jump linear systems," IEEE Transactions on Automatic Control, vol. 51, no. 11, pp. 1837-1841, 2006.

[25] J. Zhu, J. Park, K.-S. Lee, and M. Spiryagin, "Robust extended Kalman filter of discrete-time Markovian jump nonlinear system under uncertain noise," Journal of Mechanical Science and Technology, vol. 22, no. 6, pp. 1132-1139, 2008.

[26] S. K. Nguang and P. Shi, "Robust $\mathscr{\ell}_{\infty}$ output feedback control design for Takagi-Sugeno systems with Markovian jumps: a linear matrix inequality approach," Journal of Dynamic Systems, Measurement and Control, vol. 128, no. 3, pp. 617-625, 2006.

[27] J. Zhu, H.-S. Xi, H.-B. Ji, and B. Wang, "Robust adaptive tracking for Markovian jump nonlinear systems with unknown nonlinearities," Discrete Dynamics in Nature and Society, vol. 2006, Article ID 92932, 18 pages, 2006.

[28] Z. Jin, X. Hongsheng, X. Xiaobo, and J. Haibo, "Guaranteed control performance robust LQG regulator for discrete-time Markovian jump systems with uncertain noise," Journal of Systems Engineering and Electronics, vol. 18, no. 4, pp. 885-891, 2007.

[29] C. Chen, H.-X. Li, and D. Dong, "Hybrid control for robot navigation: a hierarchical Q-learning algorithm," IEEE Robotics and Automation Magazine, vol. 15, no. 2, pp. 37-47, 2008.

[30] D. Dong, C. Chen, H. Li, and T.-J. Tarn, "Quantum reinforcement learning," IEEE Transactions on Systems, Man, and Cybernetics, Part B, vol. 38, no. 5, pp. 1207-1220, 2008.

[31] H. Deng, M. Krstić, and R. J. Williams, "Stabilization of stochastic nonlinear systems driven by noise of unknown covariance," IEEE Transactions on Automatic Control, vol. 46, no. 8, pp. 1237-1253, 2001. 
[32] Z.-J. Wu, X.-J. Xie, and S.-Y. Zhang, "Adaptive backstepping controller design using stochastic smallgain theorem," Automatica, vol. 43, no. 4, pp. 608-620, 2007.

[33] I. M. Y. Mareels and D. J. Hill, "Monotone stability of nonlinear feedback systems," Journal of Mathematical Systems, Estimation, and Control, vol. 2, no. 3, pp. 275-291, 1992.

[34] B. Øksendal, Stochastic Differential Equations, Springer, New York, NY, USA, 2000.

[35] M. M. Polycarpou and P. A. Ioannou, "A robust adaptive nonlinear control design," Automatica, vol. 32, no. 3, pp. 423-427, 1996.

[36] B. Wang, H. Ji, J. Zhu, and X. Xiao, "Robust adaptive control of polynomial lower-triangular systems with dynamic uncertainties," in Proceedings of the 6th World Congress on Intelligent Control and Automation (WCICA '06), vol. 1, pp. 815-819, Dalian, China, June 2006. 